\title{
Etude des facteurs de variation de la dégradation ruminale de la matière sèche de l'ensilage de maïs
}

\author{
P Chapoutot 1, G Cabon 2, F Boudet 1, D Sauvant 1, F Gatel 3 \\ 1/NRA Nutrition et Alimentation, INA-PG, 16 rue Claude Bernard, 75231 Paris Cedex 05 ; \\ 2ITCF, Ferme de la Jaillère, La Chapelle St Sauveur, 44370 Varades , \\ 3ITCF, Pouline, 41100 Villerable, France
}

La valeur alimentaire de l'ensilage de maïs (EM) dépendrait largement de sa cinétique de dégradation dans le réticulo-rumen. Une étude, conduite sur des échantillons d'EM d'origines diverses, a donc été entreprise pour chercher à mieux comprendre les causes de variation de ces cinétiques.

23 échantillons d'EM $(5,9<$ MAT $=7,5 \%$ MS $<8,7 ; 39,0<\mathrm{NDF}=44,7 \% \mathrm{MS}<53,7$; $20,8<\mathrm{ADF}=24,0 \% \mathrm{MS}<29,3 ; 2,0<\mathrm{ADL}=$ $2,6 \% M S<3,4)$ ont fait l'objet de mesures de dégradation in situ par la méthode standardisée (Michalet-Doreau et al, 1987, Bull Tech CRZV Theix, INRA, 69, 5-7) sur 3 vaches taries fistulées du rumen recevant un régime constitué de $70 \%$ foin de pré et $30 \%$ concentré distribué en 2 repas ( $9 h$ et $17 \mathrm{~h}$ ). Les aliments, broyés à $1 \mathrm{~mm}$, ont été incubés (9 répétitions: 3 vaches $\times 3$ jours) dans des sachets de Nylon (maille : $50 \mu$, dimensions internes : $11 \times 6 \mathrm{~cm}$ ) pendant 2, 4, 8, 16, 24 et $48 \mathrm{~h}$. Après lyophilisation et pesée, les résidus ont fait l'objet de dosages ( $N \times 6,25$, NDF, $A D F$, et $A D L)$ de façon à calculer les quantités résiduelles de matière sèche (QMSr), de matières azotées (QMATr), de glucides pariétaux (QNDFr, QADFr, QADLr) et de constituants cytoplasmiques estimés par la fraction NSC (QNSCr = QMSr - QNDFr QMATr). Ces valeurs sont exprimées par rapport à la MS initiale.

La MS présente une cinétique de dégradation remarquablement linéaire ne correspondant pas à un processus du premier ordre. A $2 \mathrm{~h}$ et à $48 \mathrm{~h}$, la QMSr a varié selon les échantillons de 40,8 à $59,1 \%$ et de 15,6 à $27,0 \%$ respectivement. Dans le court terme, ces variations sont expliquées en partie par les différences de teneurs en parois de I'EM ( $R=0,70$ à $2 \mathrm{~h}$ ) qui limitent vraisemblablement la dégradation de l'ensemble de la MS par les micro-organismes du rumen. En effet, les variations de QMSr sont reliées non seulement à l'accumulation des constituants pariétaux non dégradés $(R=0,83)$ mais également à celle des MAT $(R=0,79)$ et des éléments cytoplasmiques $(R=0,62)$. Dans le long terme, les teneurs en lignocellulose et en lignine de I'EM deviennent les facteurs explicatifs principaux des écarts de QMSr (respectivement $R=0,70$ et $R=0,64$ à $48 \mathrm{~h}$ ). Lorsque la durée d'incubation s'allonge, l'influence des variations des quantités résiduelles de parois tend à s'accroître tandis que celle des constituants cytoplasmiques diminue. Ainsi à $48 \mathrm{~h}$, la QMSr dépend beaucoup plus étroitement de l'importance du NDF résiduel : ( $Q M S r=1,15$ QNDFr + 1,51; $R=0,96$; $E T R=0,51)$ que des MAT $(R=0,79)$ ou des glucides cytoplasmiques $(R=0,40)$ non dégradés.

Ces mesures révèlent d'une part le rôle d'obstacle joué dans le court terme par les parois végétales vis-à-vis de la libération dans le rumen des contenus cellulaires qui peut contribuer à expliquer certaines variations de valeur d'encombrement de l'EM, et d'autre part l'influence de la fraction non dégradable des parois végétales dans la valorisation énergétique de l'EM, déjà démontrée par Andrieu et al, 1993 (Ann Zootech, 42, 221-249). 